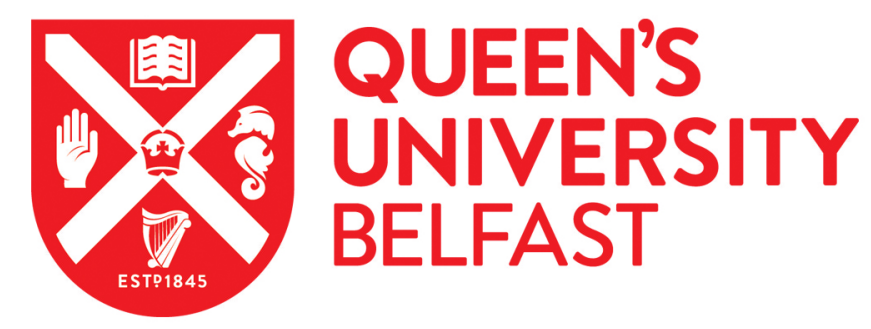

\title{
High dilution surface-enhanced Raman spectroscopy for rapid determination of nicotine in e-liquids for electronic cigarettes
}

Itoh, N., \& Bell, S. E. J. (2017). High dilution surface-enhanced Raman spectroscopy for rapid determination of nicotine in e-liquids for electronic cigarettes. The Analyst, 142, 994-998. https://doi.org/10.1039/C6AN02286C

\section{Published in:}

The Analyst

\section{Document Version:}

Peer reviewed version

Queen's University Belfast - Research Portal:

Link to publication record in Queen's University Belfast Research Portal

\footnotetext{
General rights

Copyright for the publications made accessible via the Queen's University Belfast Research Portal is retained by the author(s) and / or other copyright owners and it is a condition of accessing these publications that users recognise and abide by the legal requirements associated with these rights.
}

Take down policy

The Research Portal is Queen's institutional repository that provides access to Queen's research output. Every effort has been made to ensure that content in the Research Portal does not infringe any person's rights, or applicable UK laws. If you discover content in the Research Portal that you believe breaches copyright or violates any law, please contact openaccess@qub.ac.uk. 


\section{High dilution surface-enhanced Raman spectroscopy for rapid}

\section{2 determination of nicotine in e-liquids for electronic cigarettes}

3

$4 \quad$ Nobuyasu Itoh*a and Steven E.J. Bell ${ }^{\mathrm{b}}$

5

6 0 Japan. 5

\section{Abstract}

a National Metrology Institute of Japan (NMIJ), National Institute of Advanced Industrial Science and Technology (AIST), Central 3, 1-1-1 Umezono, Tsukuba, Ibaraki 305-8563, Japan

${ }^{\mathrm{b}}$ Innovative Molecular Materials Group, School of Chemistry and Chemical Engineering, Queen's University Belfast, David Keir Building, Stranmillis Road, Belfast BT9 5AG, United Kingdom

*Corresponding author. National Metrology Institute of Japan (NMIJ), National Institute of Advanced Industrial Science and Technology (AIST), 1-1-1 Umezono, Tsukuba, Ibaraki 305-8563,

E-mail address: nobuyasu-itoh@aist.go.jp (N. Itoh).

The rise in popularity of electronic cigarettes and the associated new legislation concerning e-liquids has created a requirement for a rapid method for determining the nicotine content of e-liquids in the field, ideally at the point of sale. Here we have developed a rapid method based on surface-enhanced Raman spectroscopy (SERS) with Au colloid and an isotopelabeled nicotine ( $\mathrm{d}_{4}$-nicotine) internal standard for measurement/quantification of samples which contain 10's of $\mathrm{mg} \mathrm{mL}^{-1}$ nicotine in a complex viscous matrix. The method is novel within the area of SERS because it uses high dilution (ca. 4000×) in the sample preparation which dilutes out the effects of the viscous glycerin/glycerol medium and any flavouring or colouring agents present but still allows for very accurate calibration with high 
reproducibility. This is possible because the nicotine concentration in the e-liquids $(\leq 24$ $\mathrm{mg} \mathrm{mL} \mathrm{m}^{-1}$ ) is several orders of magnitude above the working range of the SERS measurement. The method has been tested using a portable Raman spectrometer and a very large set of 42 commercial e-liquids to check there is no matrix interference associated with

\section{Introduction} different manufacturers/flavourings/colouring agents etc. Finally, as an alternative to determining the nicotine concentration by measuring peak heights in the spectra, the concentration was also estimated by comparing the sample spectra with those of a set of standard sample which prepared at known concentrations and held in a spectral library file in the spectrometer. This simple approach allows concentration to be estimated without any complex data analysis and lends itself readily to handheld Raman system which are typically designed to carry out library searching using the internal software for materials identification. Library searching against standards correctly classified 41 of the 42 test liquids as belonging to the correct concentration group. This high dilution SERS approach is suitable for analysis of sample types that have reasonably high concentrations of analytes but suffer from matrix problems and it therefore has broad potential for applications across the food, pharmaceutical and nutraceutical areas.

Electronic nicotine delivery systems, commonly called electronic cigarettes (ECs), are battery-powered devices that simulate tobacco cigarettes by converting nicotine-containing liquid into an aerosol. ECs have gained popularity in the past few years, primarily among smokers who want to reduce the risks of smoking because ECs do not produce the numerous chemicals found in conventional tobacco smoke. ${ }^{1-3}$ ECs use e-liquids which contain nicotine, flavouring/colouring components and a base such as propylene glycol, glycerin, or a mixture of these two substances. The nicotine concentrations of available e-liquids typically range from $0 \mathrm{mg} \mathrm{mL}^{-1}$ to $24 \mathrm{mg} \mathrm{mL}^{-1}$ and 
51 numerous different flavours are available, ranging from tobacco flavours (which are similar to cigarettes) to menthol, fruits and coffee. ${ }^{4-7}$

Because the nicotine contained in e-liquids is both addictive and toxic, ${ }^{8}$ some countries have banned/regulated the use of ECs, e-liquids containing nicotine. ${ }^{9}, 10$ This has created a requirement for analytical methods which can be used to determine the nicotine concentrations in eliquids. The production and labelling of many of these products is not regulated at source so independent methods are required by authorities who have a legal duty to enforce legislation for public health or taxation reasons. This could be, for example, detecting nicotine in supposedly nicotine-free e-liquids or checking that the e-liquids actually contain the concentrations of nicotine stated on their containers by the manufacturers. ${ }^{5,}$, $11-13$

Nicotine concentrations in e-liquids have been widely quantified by gas chromatography (GC) or high-performance liquid chromatography (HPLC). Sample solutions for these instruments are commonly prepared by the pipetting of e-liquids followed by dilution/extraction and are mixed with/without internal standards such as quinoline. ${ }^{4-7,}$ 11-15 These methods are well-established and accurate but they are time-consuming (usually more than 30 min for each sample) and not suitable for rapid field testing at point of sale.

While conventional vibrational spectroscopy has some of the aspects required for field testing, such as portability and acceptable cost, the nature of the sample makes conventional vibrational analysis of e-liquids difficult. For IR the aqueous/glycerol medium will interfere while the nicotine concentration is too low for normal Raman analysis, moreover the samples can give strong fluorescence backgrounds with common excitation wavelengths. In principle, surfaceenhanced Raman spectroscopy SERS should have appropriate sensitivity but there are potential problems due to the oily and highly viscous nature of the e-liquids (propylene glycol: $40.4 \mathrm{mPa} \bullet \mathrm{s}$ at

$7425^{\circ} \mathrm{C}$; vegetable glycerin: $934 \mathrm{mPa} \cdot \mathrm{s}$ at $\left.25^{\circ} \mathrm{C}\right)^{16}$ which could hinder aggregation and also interfere with adsorption of the analyte to the enhancing surface. In addition, the numerous different colouring/flavouring compounds can also potentially give their own interfering SERS signals. ${ }^{17}$ 
Here we show that these problems in the SERS analysis can be overcome because the

sensitivity of SERS is vastly better than is required to detect the analyte in the unprocessed samples. Literature data has shown SERS nicotine detection at the low ppm level ${ }^{18-21}$ while the e-liquids are 4 orders of magnitude higher. This means the samples can be diluted down dramatically, which removes problems associated with the glycerin/glycerol medium and similarly reduces the flavouring compounds to undetectably low concentrations. This has allowed us to develop a convenient procedure for nicotine screening in e-liquids suitable for field use which combines high dilution in the sample preparation with very straightforward data analysis that can be carried out on simple portable Raman instruments where sample spectra are automatically compared to a library of standard spectra of samples prepared at different concentrations.

\section{Experimental}

\section{Chemicals and samples}

Nicotine, deuterium-labeled nicotine ( $\mathrm{d}_{4}$-nicotine), and magnesium sulfate $\left(\mathrm{MgSO}_{4}\right)$ were obtained from Sigma-Aldrich (St. Louis, MO, USA). The Au colloid (particle size: $50 \mathrm{~nm}, 4.50 \times$ $10^{10}$ particles $\mathrm{mL}^{-1}$ ) solution was obtained from BBI solutions (Cardiff, UK). Monopropylene glycol (PG, Pharma grade) and vegetable glycerin (VG, USP Kosher grade) were obtained from Classikool (Essex, UK). Deteriorated nicotine was a sample of pure nicotine which had been stored for more than 10 years at room temperature in the reagent cabinet of our laboratory.

E-liquid solutions were obtained from manufacturers in the United Kingdom (Table S1). A set of samples comprising eight flavours, each at 4 different nicotine levels were purchased so the calibration could be tested with a range of flavours. A further set of 10 assorted liquids with different flavours were also used to allow the influence of colourings and flavours as well as different types of bases to be examined over a broad range of liquid types. All of the e-liquids obtained were stored at room temperature in the dark. 


\section{Preparation of solutions}

Nicotine reagents were diluted with double distilled water (DDI). To avoid pipetting the viscous liquid, a fixed amount, approximately $200 \mu \mathrm{L}$, of nicotine solution or e-liquid was measured by pouring it into the upturned cap of a $2 \mathrm{~mL}$ shell vial until the cap was full. The nicotine solution was transferred to a glass vial, which was previously filled with $25 \mathrm{~mL}$ of DDI (Solution A). Then, $20 \mu \mathrm{L}$ of solution A was transferred to another $1 \mathrm{~mL}$ glass vial that contained $600 \mu \mathrm{L}$ of $0.01 \mathrm{mM} \mathrm{d}_{4-}$ nicotine (Solution B). Finally, $20 \mu \mathrm{L}$ of solution B was transferred to another $1 \mathrm{~mL}$ glass vial that contained $180 \mu \mathrm{L}$ of Au colloid solution. Nicotine solutions and e-liquids were diluted ca. 4000 times throughout this preparation process. $50 \mu \mathrm{L}$ of $0.1 \mathrm{M} \mathrm{MgSO}_{4}$ was added to aggregate the colloids before their SERS spectra were recorded with a portable Raman spectrometer. The overall procedure is illustrated in Fig. S1.

\section{Measurement and classification}

The aggregated solutions were analyzed with a portable Raman spectrometer (ReporteR,

DeltaNu, WY, USA). The laser wavelength was $785 \mathrm{~nm}$, and the spectral range $200 \mathrm{~cm}^{-1}$ to 2000 $\mathrm{cm}^{-1}$. Spectrometer $\mathrm{cm}^{-1}$ was calibrated with a standard polystyrene accessory. The acquisition time was 2 sec x 3 accumulations. The data were analyzed with NuSpec software and no subtraction of background spectra was carried out.

For the classification of nicotine levels by library matching, the SERS spectra of mixtures of nicotine/internal standard at the appropriate concentrations $\left(0 \mathrm{mg} \mathrm{mL}^{-1}, 6 \mathrm{mg} \mathrm{mL}^{-1}, 12 \mathrm{mg} \mathrm{mL}^{-1}\right.$, $18 \mathrm{mg} \mathrm{mL}^{-1}, 24 \mathrm{mg} \mathrm{mL}^{-1}$ and $30 \mathrm{mg} \mathrm{mL}^{-1}$ ) were recorded and then used to create a small spectral library using the instrument's internal NuSPec software. To test the nicotine levels of e-liquids the spectra of the liquids prepared in the standard way with internal standard were recorded and searched against the library of standard mixtures. The nicotine concentration of the e-liquid was then estimated as being the same as that of the standard library spectrum which gave the closest match. The search used the instrument's full range spectra $\left(200 \mathrm{~cm}^{-1}\right.$ to $\left.2000 \mathrm{~cm}^{-1}\right)$ and the proprietary software which 


\section{Results and discussion}

Quantifying the nicotine content of e-liquids using normal Raman scattering measurements is extremely difficult since the spectra are dominated by signals from the propylene glycol and 134 vegetable glycerin solvent, rather than the much lower concentration ( $\mathrm{mg} \mathrm{mL}^{-1}$ ) nicotine component (Figs. S2, S3, S4). Similarly, it is not possible to increase the intensity of the nicotine bands in the spectra of undiluted e-liquids using SERS since the addition of the e-liquids prevents colloid aggregation, as shown by the observation that even the background signals from the colloid's organic stabilizing layer (which are readily detectable with simple aggregated colloid) disappear in samples prepared with undiluted e-liquids (see Fig. S5). In contrast, SERS of highly diluted e-liquids and nicotine samples was much more successful since it removed problems with aggregation, allowing

141 the nicotine to be preferentially enhanced and therefore detected, even in the presence of the other components in the e-liquid.

\section{Influence of the nicotine freshness on the Raman spectrum}

One potential problem for nicotine analysis either by Raman or SERS methods is that

nicotine decomposes in air, turning from a very pale brown to a much darker brown liquid. ${ }^{22,23}$ In this study nicotine that had been stored for more than 10 years and was very dark brown (see Fig. 1a) was tested alongside fresh nicotine to determine the effects of deterioration on the Raman and SERS spectra. As shown in Fig. 1, the Raman spectrum of the fresh nicotine showed its characteristic peaks originating from the stretching vibrations of the pyridine ring at $1027 \mathrm{~cm}^{-1}$, whereas deteriorated nicotine showed only broad emission due to fluorescence. In contrast, when the fresh and deteriorated nicotine were diluted to $10 \mu \mathrm{M}$, their SERS spectra were indistinguishable (Fig. 1b). Even though the SERS spectra of the fresh and deteriorated samples were the same, it was useful to check that deterioration did not create products that interfered with the magnitude of the 
signals e.g. by blocking surface sites. Fig. 2 shows the changes in the signal intensity of nicotine at $1027 \mathrm{~cm}^{-1}$ between 0 and $2 \mathrm{mM}$. The signal intensity of fresh nicotine increased dramatically up to $50 \mu \mathrm{M}$ and then plateaued. The slight decrease in the signal intensity at $2 \mathrm{mM}$ might be due to the reduction of colloid aggregation caused by the presence of excess nicotine in the solution. Deteriorated nicotine also showed an increase in the signal intensity with concentration up to $50 \mu \mathrm{M}$. However, the signal intensity dramatically decreased with a further increase in the concentration, and it became less than half of the maximum intensity at $200 \mu \mathrm{M}$. This was presumably due to selfabsorption of the excitation laser and Raman scattering by the dark-coloured deteriorated solutions, although it is also possible that the affinity of deteriorated nicotine may be different from those of fresh nicotine at higher concentrations ( $>50 \mu \mathrm{M})$. Nonetheless, up to $50 \mu \mathrm{M}$, as shown in Fig. $2 \mathrm{~b}$, not only were the signal intensities of both nicotine samples comparable but both also showed almost a linear relationship with concentration. Thus, we considered that both fresh and deteriorated nicotine can be quantified comparably in the range from 0 to $50 \mu \mathrm{M}$. These results are important because the with library data (see below). 
181 nicotine (from 0 to $40 \mu \mathrm{M}$ nicotine with $10 \mu \mathrm{M} \mathrm{d}_{4}$-nicotine). The signal intensity of $\mathrm{d}_{4}$-nicotine at 994 $182 \mathrm{~cm}^{-1}$ is distinct from that of nicotine at $1027 \mathrm{~cm}^{-1}$ and grows as expected with increasing nicotine 183 concentration. For quantitation, the ratio of the peak heights due to nicotine and $\mathrm{d}_{4}$-nicotine at 1027 184 and $994 \mathrm{~cm}^{-1}$, respectively, were measured. Over the range $0-40 \mu \mathrm{M}$ nicotine the reproducibility was 185 good (<5\% relative standard deviation at each concentration over the range examined), however this 186 decreased noticeably at $50 \mu \mathrm{M}$, possibly due to the influence of nicotine's small signal intensity at $187994 \mathrm{~cm}^{-1}$ and saturation effects, so the calibration range was limited to $0-40 \mu \mathrm{M}$. Over this range the 188 calibration is excellent, the plot of relative signals versus relative concentration is liner with an 189 intercept at 0.06 and $r^{2}=0.9996$, so that SERS is clearly suitable for quantification of nicotine in 190 aqueous solution.

E-liquids are quite difficult to pipette and disperse in exact volumes because they are typically oily and highly viscous ${ }^{16}$ Furthermore, the concentration range of SERS that is applicable 193 for the reliable quantification of nicotine is limited (from 0 to $40 \mu \mathrm{M}$ ). To overcome these problems, 194 we developed an easy sample preparation process using the internal volume of vial caps (see 195 Experimental and Fig. S1). This preparation process avoids accurate pipetting of e-liquids and 196 involves just mixing with DDI and other aqueous solutions, resulting in aqueous solutions containing 197 d $\mathrm{d}_{4}$-nicotine and Au colloid. This sample preparation process takes only a few minutes.

To test the efficacy of this method for e-liquids rather than aqueous nicotine solutions, the 199 nicotine concentration in tobacco flavoured e-liquids was measured. Among the examined e-liquids 200 at $0,6,12$, and $18 \mathrm{mg} \mathrm{mL}^{-1}$, the relative standard deviations in quintuplicated analyses through the 201 whole process were $2.2 \%$ for $6 \mathrm{mg} \mathrm{mL}^{-1}, 5.0 \%$ for $12 \mathrm{mg} \mathrm{mL}^{-1}$, and $4.3 \%$ for $18 \mathrm{mg} \mathrm{mL}^{-1}$, this 202 repeatability is comparable to that for pure aqueous solutions, so there were no problems in extending 203 the measurements using this technique to real e-liquids. samples) and also for another 10 flavours at $11 \mathrm{mg} \mathrm{mL}^{-1}$ to examine its ability to obtain nicotine concentrations both at different nicotine concentrations and with different interfering flavours, 
colourings and bases (Table S1). Fig. 4 shows the results of nicotine quantification in real e-liquids 208 obtained from measurements of the relative peak heights of nicotine and $\mathrm{d}_{4}$-nicotine in their spectra.

209 Because the repeatability of this method was good over this range, as discussed above, we applied 210 only duplicate analyses for each sample. Analytical results of the nicotine concentrations in all of the 211 e-liquids were comparable to those shown on their containers in samples with different nicotine 212 concentrations $\left(0 \mathrm{mg} \mathrm{mL}^{-1}:-0.4-0.0 \mathrm{mg} \mathrm{mL}^{-1}, 6 \mathrm{mg} \mathrm{mL}^{-1}: 5.7-7.2 \mathrm{mg} \mathrm{mL}^{-1}, 12 \mathrm{mg} \mathrm{mL}^{-1}: 11.2-13.3\right.$ $213 \mathrm{mg} \mathrm{mL}{ }^{-1}, 18 \mathrm{mg} \mathrm{mL}^{-1}: 17.2-18.6 \mathrm{mg} \mathrm{mL}^{-1}$, and $11 \mathrm{mg} \mathrm{mL}^{-1}: 8.5-11.7 \mathrm{mg} \mathrm{mL}^{-1}$ ), various flavours, 214 different colors and different types of bases (Fig. S6 and Table S1). This fact suggests that the 215 analytical results obtained by this method are free from interference due to flavours, colourings and 216 types of base.

Finally, the portable Raman system used in this study can automatically compare newly acquired spectra with library data in real time. This function becomes possible with $\mathrm{d}_{4}$-nicotine 219 addition and is very convenient for rapidly estimating the approximate nicotine content, a task which is made easier by the fact that most of the available e-liquids contain nicotine levels which vary in 221 multiples of 6 , such as $0,6,12$, and $18 \mathrm{mg} \mathrm{mL}^{-1} \cdot{ }^{4-7}$ Here the SERS spectral data from the calibration curve was used to build a spectral library that the spectra for each e-liquid could be compared against. This allowed the nicotine level in the e-liquids to be classified by finding which spectrum in the library they matched most closely. Fig. 4 shows the classification of the nicotine levels in all 42 samples obtained by library matching, along with the results from the quantitative analysis. In the plot, the shape of each of the points is used to indicate which of the 5 concentration values the library matching gave. The approach was remarkably successful, only 1 of the 42 samples was incorrectly classified and in that case the sample was classified as belonging the nearest neighbor (actual $11 \mathrm{mg}$ $\mathrm{mL}^{-1}$, estimated value $6 \mathrm{mg} \mathrm{mL}^{-1}$ ). This level of accuracy also meant that the method allowed samples 230 which contained nicotine to be distinguished from those that did not with confidence (Tables S2 and 231 S3). 


\section{Conclusions}

We have developed a new method for the screening of nicotine in e-liquids which combines

235 an easy sample preparation process with SERS and a portable Raman spectrometer. The method can 236 be used either for full quantitation of nicotine concentration or for rapid estimation of the nicotine 237 level by library matching. Importantly, the results are not affected by flavours, colourings, type of 238 base or the freshness of the nicotine. This was possible because the high sensitivity of SERS meant 239 that the sample could be significantly diluted (ca. 4000×) in the sample preparation which diluted out 240 matrix effects from the glycerol present and also reduced interference from flavouring and colouring 241 compounds below detectable levels. This approach of combining high sample dilution with SERS 242 clearly has the potential to be applied to other sample types where matrix effects may be significant, 243 such as foodstuffs or topical pharmaceuticals. 


\section{Notes and references}

248 1. P. Hajek, J.-F. Etter, N. Benowitz, T. Eissenberg and H. McRobbie, Addiction, 2014, 109, 249 1801-1810.

$250 \quad 2 . \quad$ J.-F. Etter and C. Bullen, Addiction, 2011, 106, 2017-2028.

251 3. J.-F. Etter, BMC Public Health, 2010, 10, 231.

252 4. M. L. Goniewicz, R. Gupta, Y. H. Lee, S. Reinhardt, S. Kim, B. Kim, L. Kosmider and A. 253 Sobczak, Int. J. Drug Policy, 2015, 26, 583-588.

254 5. B. Davis, M. Dong, J. Kim and P. Talbot, Nicotine Tob. Res., 2015, 17, 134-141.

255 6. M. L. Goniewicz, T. Kuma, M. Gawron, J. Knysak and L. Kosmider, Nicotine Tob. Res., 2013, $256 \quad 15,158-166$.

257 7. J.-F. Etter, E. Zäther and S. Svensson, Addiction, 2013, 108, 1671-1679.

$258 \quad$ 8. B. Mayer, Arch. Toxicol., 2014, 88, 5-7.

259 9. J. E. Henningfield and G. S. Zaatari, Tob. Control, 2010, 19, 89-90.

260 10. W. H. Organization, Conference of the Parties to the WHO framework convention on tobacco 261 control, 2014, FCTC/COP/6/10 Rev.1, 13 pp. (last accessed: August 9, 2016)

262 11. J. G. Lisko, H. Tran, S. B. Stanfill, B. C. Blount and C. H. Watson, Nicotine Tob. Res., 2015, $263 \quad 17,1270-1278$.

264 12. J. M. Cameron, D. N. Howell, J. R. White, D. M. Andnyak, M. E. Layton and J. M. Roll, Tob. $265 \quad$ Control, 2014, 23, 77-78.

266 13. T. Cheng, Tob. Control, 2014, 23, ii11-ii17.

267 14. M. Famele, C. Ferranti, C. Abenavoli, L. Palleschi, R. Mancinelli and R. Draisci, Nicotine 268 Tob. Res., 2015, 17, 271-279.

269 15. M. L. Trehy, W. Ye, M. E. Hadwiger, T. W. Moore, J. F. Allgire, J. T. Woodruff, S. S. Ahadi, 270 J. C. Black and B. J. Westenberger, J. Liq. Chromatogr. Related Technol., 2011, 34, $1442-$ $271 \quad 1458$.

272 16. CRC Handbook of Chemistry and Physics, CRC Press, 89th edn., 2009. 
273 17. E. C. L. Ru and P. G. Etchegoin, Principles of Surface-Enhanced Raman Spectroscopy, $274 \quad$ Elsevier, Amsterdam, 2009.

$275 \quad$ 18. S. E. J. Bell and N. M. S. Sirimuthu, Analyst, 2004, 129, 1032-1036.

276 19. J. Jung, J. Choo, D. J. Kim and S. Lee, Bull. Korean Chem. Soc., 2006, 27, 277-280.

277 20. M. B. Mamián-López and R. J. Poppi, Anal. Chim. Acta, 2013, 760, 53-59.

278 21. O. Alharbi, Y. Xu and R. Goodacre, Analyst, 2014, 139, 4820-4827.

279 22. H. Schievelbein, Beitr. Tabakforsch., 1962, 199-274.

280 23. Centers for Disease Control and Prevention, Nicotine: Systemic agent. Available at: 281 http://www.cdc.gov/niosh/ershdb/emergencyresponsecard_29750028.html (last accessed: $282 \quad$ August 9, 2016).

283 24. A. Mechlińska, L. Wolska and J. Namieśnik, Trend Anal. Chem., 2010, 29, 820-831. 284 285 286 

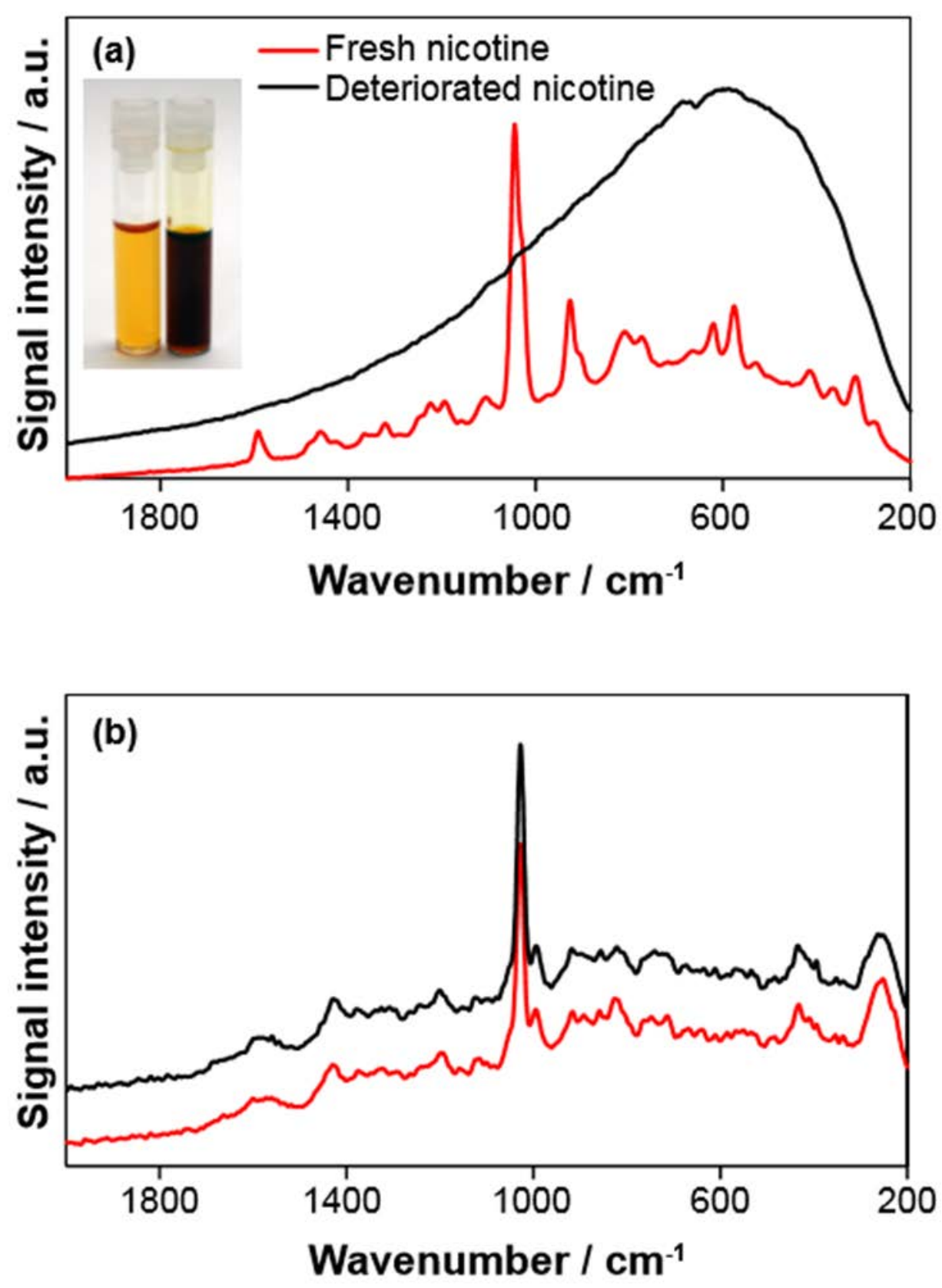

Fig. 1

(a) Raman spectra of fresh and deteriorated nicotine. The inset photograph shows fresh (left) and deteriorated (right) nicotine in glass vials. (b) SERS spectra of fresh and deteriorated nicotine. 

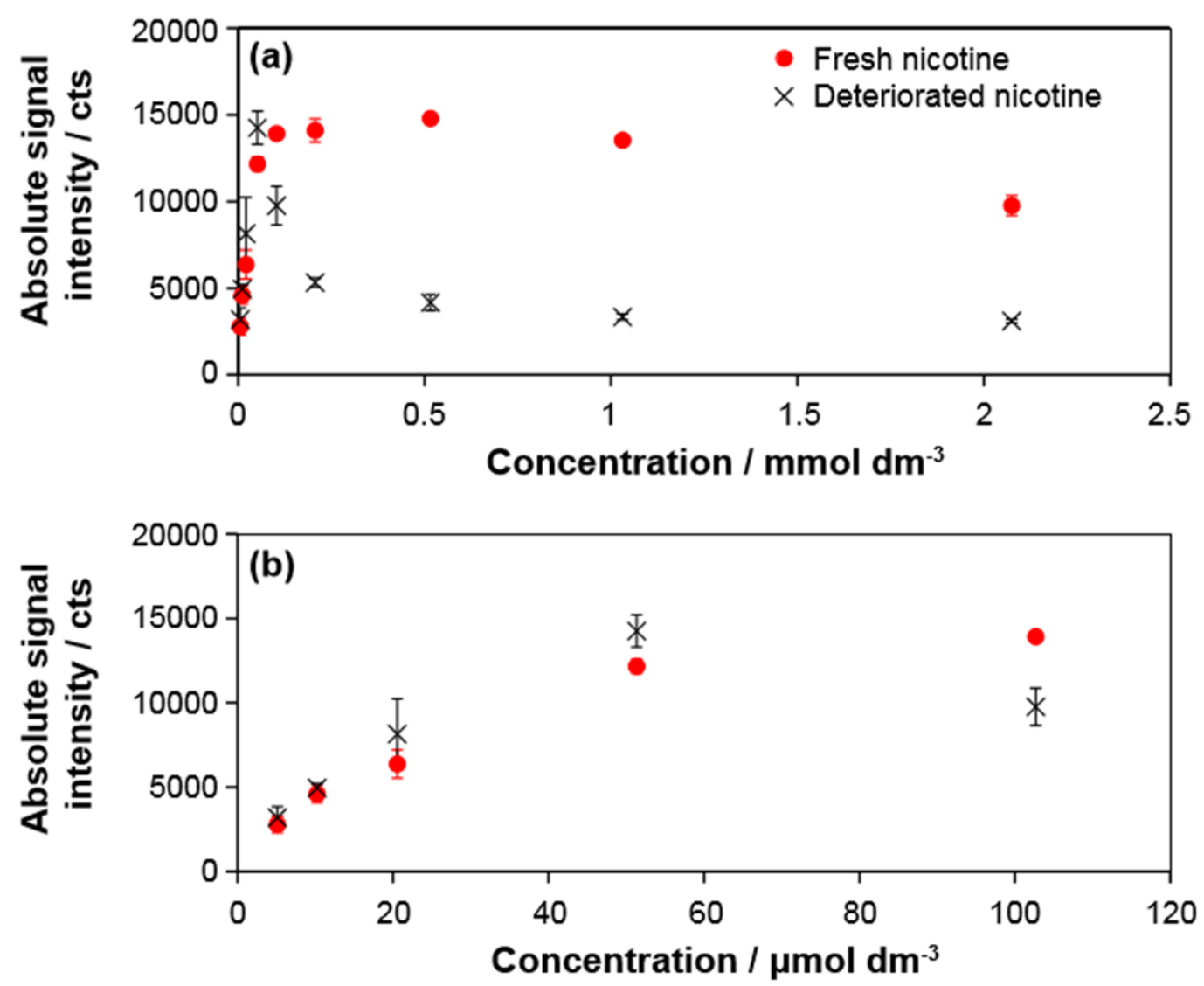

Fig. 2

299 Plot of the intensity of the SERS band at $1027 \mathrm{~cm}^{-1}$ and nicotine concentration (a) from 0 to $2 \mathrm{mmol}$ $300 \mathrm{~L}^{-1}$ and (b) magnified between 0 to $100 \mu \mathrm{mol} \mathrm{L} \mathrm{L}^{-1}$. Data for fresh and deteriorated nicotine are shown. 301 The error bars indicate the standard deviation in triplicate analyses. 

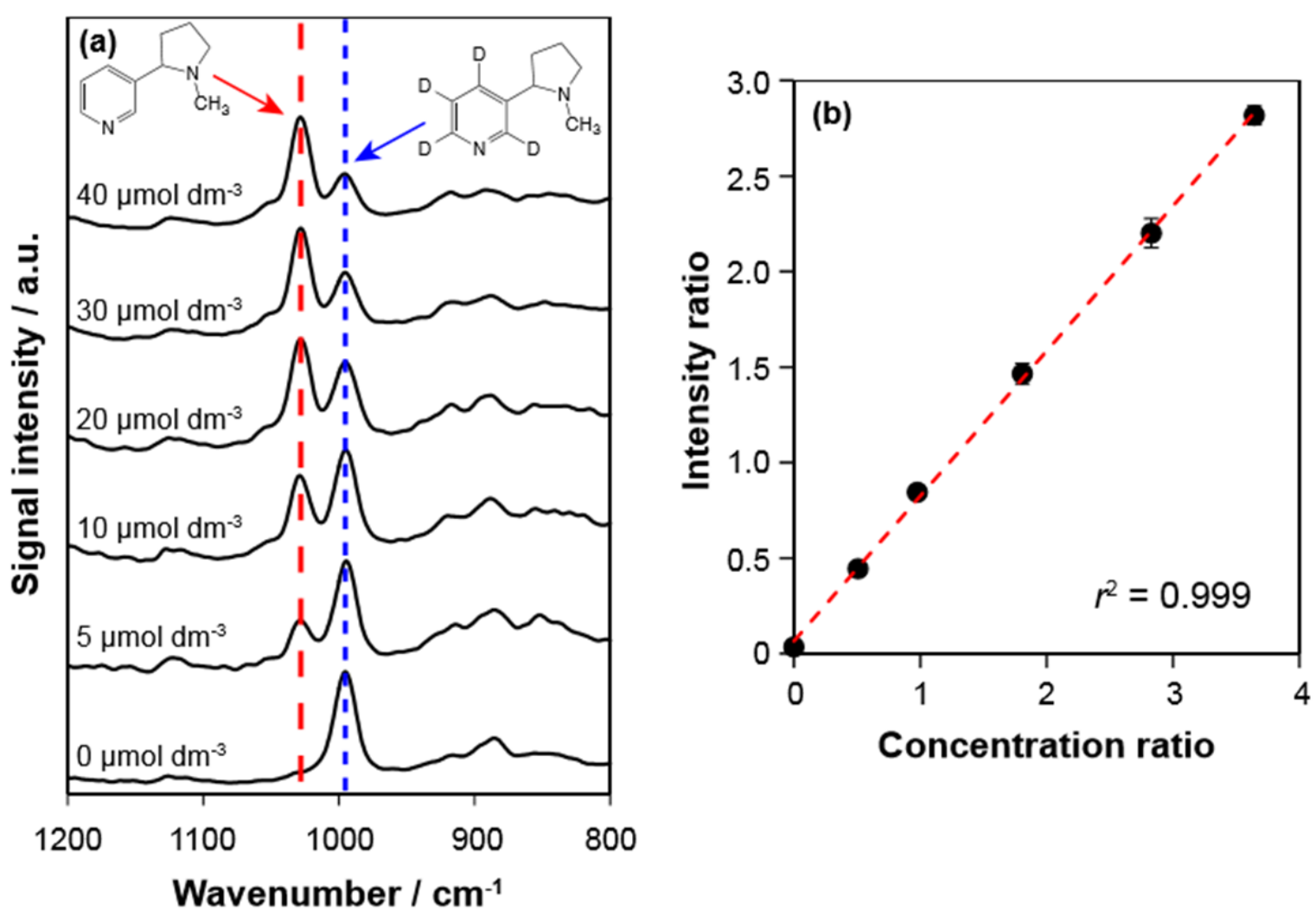

$312 \quad$ Fig. 3

313 (a) Changes in the SERS spectra of a nicotine $\left(1027 \mathrm{~cm}^{-1}\right)$ and $\mathrm{d}_{4}$-nicotine $\left(994 \mathrm{~cm}^{-1}\right)$ mixture in 0 to $31440 \mu \mathrm{M}$ nicotine solutions with $\mathrm{d}_{4}$-nicotine internal standard fixed at $10 \mu \mathrm{M}$. Inset shows the structures 315 of nicotine and $\mathrm{d}_{4}$-nicotine. (b) Plot of the concentration ratio of nicotine and $\mathrm{d}_{4}$-nicotine against the 316 ratio of their characteristic $\left(1027: 994 \mathrm{~cm}^{-1}\right)$ bands. Error bars indicate the standard deviation in 317 quintuplicate analyses. 


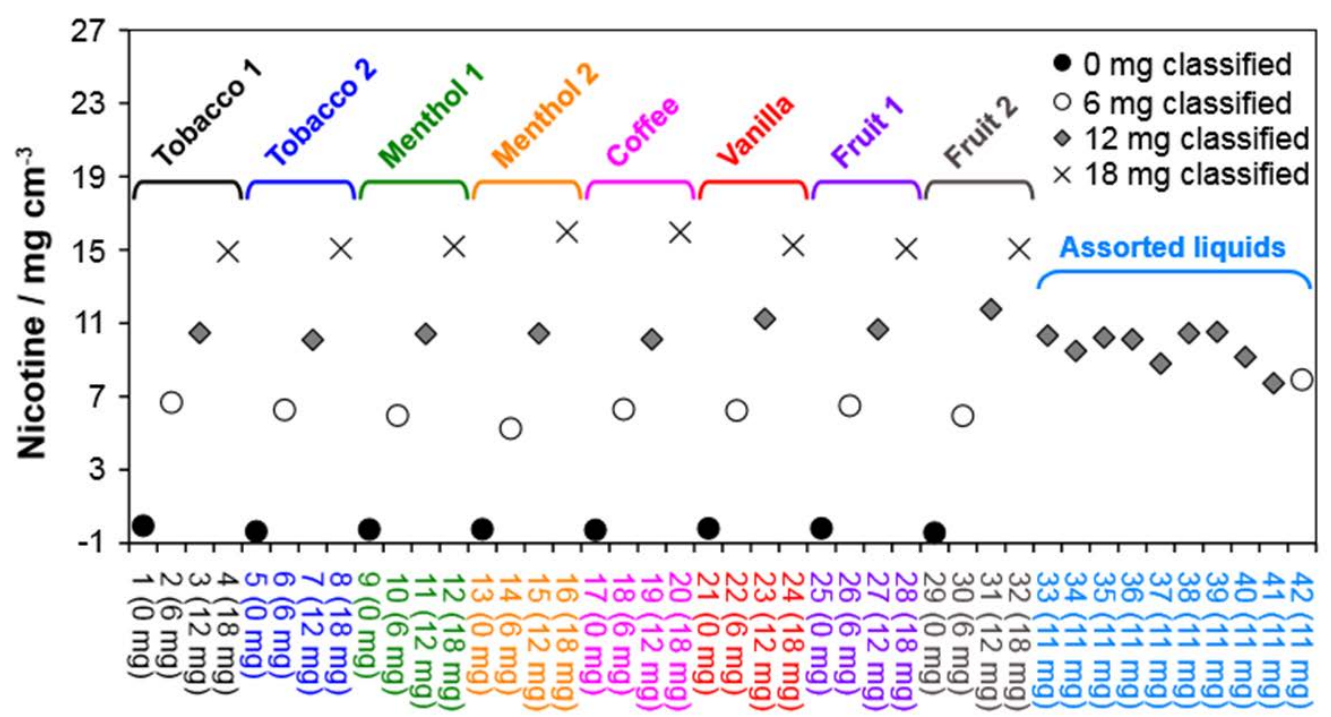

Sample No.

Fig. 4

328 Plot illustrating the results of nicotine SERS analysis in commercial e-liquids. Values in parentheses 329 on the $\mathrm{x}$-axis are the nicotine concentrations shown on each container. The positions of the points 330 show the analytical values obtained by measuring relative peak heights of nicotine and $\mathrm{d}_{4}$-nicotine 331 for each of the samples. The symbols used to mark the points indicate which standard spectrum the 332 unprocessed sample spectra matched in the spectral library. These latter values can be used to estimate 333 the nicotine concentration without explicitly measuring peak heights in the spectra. 